\title{
Oblique breathers generated by a flow of two-component Bose-Einstein condensate past a polarized obstacle
}

\author{
A. M. Kamchatnov ${ }^{1}$ and Y. V. Kartashov ${ }^{1}$ \\ ${ }^{1}$ Institute of Spectroscopy, Russian Academy of Sciences, Troitsk, Moscow, 142190, Russia

\begin{abstract}
We predict that oblique breathers can be generated by a flow of two-component Bose-Einstein condensate past a polarized obstacle which attracts one component of the condensate and repels the other one. The breather exists if intra-species interaction constants differ from the inter-species interaction constant and it corresponds to the nonlinear excitation of the so-called polarization mode with domination of the relative motion of the components. Approximate analytical theory is developed for the case of small-amplitude breathers that is in reasonable agreement with the exact numerical results.
\end{abstract}

PACS numbers: 03.75.Kk,03.75.Mn

Introduction.-Flow of Bose-Einstein condensates (BECs) past obstacles reveals a number of different nonlinear excitations of the condensate. For example, in a one-component condensate with the sound velocity $c_{s}$, whose value is determined by the background density, nonlinear interaction constant and the mass of atoms, the flow is superfluid for velocities $V$ satisfying the condition $M \equiv V / c_{s}<M_{c}\left(M_{c} \approx 0.37\right.$ for the flow past a disk in 2D geometry). For greater Mach numbers $M>M_{c}$ vortices are generated by the flow what means loss of superfluidity (see [1 [5]). Another channel of dissipation opens, according to the Landau criterion, when the flow velocity exceeds the sound velocity, that is at $M=1$ (see, e.g., [6, 7]). In this case, the interference of Bogoliubov waves generated by a supersonic flow leads to formation of a so-called "ship-wave" pattern located outside the Mach cone [8, 9]. Inside the Mach cone the vortex streets are generated in the flow velocity interval $1<M<1.44$, but for velocities with $M>1.44$ very specific oblique dark solitons [10] are generated [11 13]. They have been observed in experiments 14, 15] with flows of polariton condensates past obstacles. These nonlinear structures manifest themselves as dips in the distributions of the condensate's density.

Creation of two-component atomic [16, 17] and spinor polariton [18, 19] condensates triggered extensive research activity, both theoretical and experimental, centered around nonlinear properties of two-species superfluids (see review articles [20,21 and references therein). One of new properties of such superfluids is a possibility of formation of topological excitations 22, 23. Another specific feature of two-species superfluids is the existence of two modes of motion which can be called density waves and polarization waves - in the density waves the two species move mainly in phase with each other whereas in the polarization waves they move mainly in counter phase. In the linear limit there exist, correspondingly, two types of sound waves which in problems like a description of wave patterns generated by the flow past an obstacle define two Mach cones. Consequently, two types of ship waves are generated by the flow of a two- component condensate past an obstacle. The existence of two different types of excitations in two-component condensate suggests the possibility of two different types of oblique dark solitons that may be generated upon the interaction of condensate with a defect. However, previously only one type of oblique dark solitons 24] have been observed in the flow past a non-polarized obstacle, i.e. the obstacle whose potential acts equally on both species of the condensate. In this case the potential disturbs both species "in phase", that is the symmetry of the potential coincides with the symmetry of the density waves, and only such waves are excited by the flow. This suggests that another, previously elusive, polarization mode can be generated by a polarized obstacle which acts differently on different species of the condensate.

In this Letter we study the properties of wave patterns generated by the two-dimensional flow of a twocomponent condensate past such a polarized obstacle and demonstrate that in this case a previously unknown type of excitations enter the scene-oblique breather. One of the most distinctive features of such breathers is that in contrast to conventional dark-dark solitons generated by non-polarized potentials, the density distributions in two components of the breather have different shapes with deep out-of-phase modulation. The properties of oblique breathers are studied numerically in the framework of a system of two coupled Gross-Pitaevskii (GP) equations and they are explained analytically in the smallamplitude limit by reducing the GP system to a mKdV equation for a weakly nonlinear polarization mode.

The model. - In the mean field theory the dynamics of two-component BEC is described by the system of GP equations in standard non-dimensional form

$$
\begin{aligned}
& i \frac{\partial \psi_{1}}{\partial t}+\frac{1}{2} \Delta \psi_{1}-\left(g_{11}\left|\psi_{1}\right|^{2}+g_{12}\left|\psi_{2}\right|^{2}\right) \psi_{1}=\sigma_{1} U(\mathbf{r}) \psi_{1}, \\
& i \frac{\partial \psi_{2}}{\partial t}+\frac{1}{2} \Delta \psi_{2}-\left(g_{12}\left|\psi_{1}\right|^{2}+g_{22}\left|\psi_{2}\right|^{2}\right) \psi_{2}=\sigma_{2} U(\mathbf{r}) \psi_{2},
\end{aligned}
$$

where Laplacian $\Delta$ acts on two spatial coordinates $\mathbf{r}=$ $(x, y)$. We assume that particles in both species have 
the same mass and the potential $\sigma_{k} U(\mathbf{r}, t)$ of the obstacle is repulsive if $\sigma_{k}=1$ and attractive if $\sigma_{k}=-1$. In our numerical simulations it is modeled by the form $U(\mathbf{r})=U_{0} \exp \left(-\mathbf{r}^{2} / a^{2}\right)$ with $U_{0}=1.0, a=2$. The nonlinear interaction constants $g_{i k}$ are supposed to be positive. For simplicity we assume that both components have in an undisturbed uniform state the same densities $\rho_{1}=\rho_{2}=\rho_{0} / 2$, where $\rho_{1}=\left|\psi_{1}\right|^{2}, \rho_{2}=\left|\psi_{2}\right|^{2}$, and $\rho_{0}$ is an undisturbed total density $\rho=\rho_{1}+\rho_{2}$ at $|\mathbf{r}| \rightarrow \infty$.

Linearization of the system (1) for slightly disturbed background state yields the dispersion relations for the linear waves in the two-component condensate (see, e.g., [24]) $\omega_{d, p}^{2}=c_{d, p}^{2} k^{2}+\frac{1}{4} k^{4}$, where

$$
c_{d, p}=\frac{1}{2}\left[\rho_{0}\left(g_{11}+g_{22} \pm \sqrt{\left(g_{11}-g_{22}\right)^{2}+4 g_{12}^{2}}\right)\right]^{1 / 2}
$$

are the velocities of the density $\left(c_{d}\right.$, upper sign) and polarization $\left(c_{p}\right.$, lower sign) waves, while $\omega_{d, p}$ are the frequencies describing temporal evolution of perturbations $\propto \exp \left(-i \omega_{d, p} t\right)$. The presence of two different velocities leads to the existence of two Mach cones defined by the relations

$$
\sin \chi_{d, p}=\frac{c_{d, p}}{V} \equiv \frac{1}{M_{d, p}},
$$

where $M_{d, p}=V / c_{d, p}$ are the corresponding Mach numbers; $\chi_{d, p}$ are the angles between the direction of the flow and the lines representing the density and polarization cones.

Oblique breathers.- In order to demonstrate the principal difference between wave patterns generated by nonpolarized $\left(\sigma_{1}=\sigma_{2}=1\right)$ and polarized $\left(\sigma_{1}=-\sigma_{2}=1\right)$ obstacles, we have solved the system (1) numerically for these two cases using the input conditions $\psi_{1,2}=$ $\left(\rho_{0} / 2\right)^{1 / 2} \exp (i V x)$. Typical results are illustrated in Fig. 1. For the non-polarized obstacle the density ship waves are located outside the density Mach cone and a dark-dark soliton is located inside it. Remarkably, the existence of the polarization Mach cone is not manifested at all in the density distributions of both condensate components - linear and nonlinear polarization waves are not excited by the non-polarized obstacle. In sharp contrast, the polarized obstacle leads to much richer dynamics and generates both density ship waves (outside the outer Mach cone) and polarization ship waves (outside the inner Mach cone). The density waves oscillate in phase in both components what increases the amplitude of oscillations in the total density, whereas the counterphase oscillations in the condensate components in the polarization ship waves lead to cancelation of oscillations in the total density. The polarized obstacle does not excite a usual dark-dark soliton, but instead a more complicated structure is generated in the vicinity of the polarization Mach cone. We shall call this structure an oblique breather since, as we shall see below, it can be related

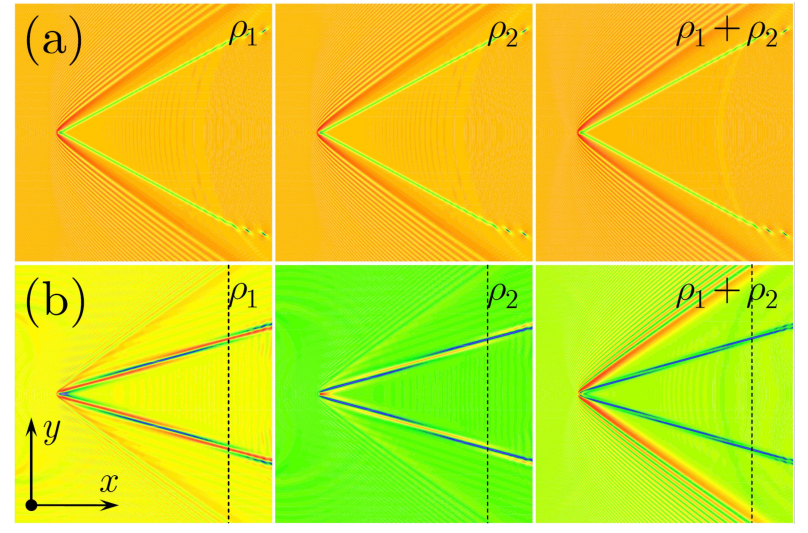

Figure 1. (Color online.) Distributions of the densities of the first (left column) and second (central column) components, as well as of total density (right column) for (a) non-polarized obstacle with $\sigma_{1}=\sigma_{2}=1$, and for (b) polarized obstacle with $\sigma_{1}=1, \sigma_{2}=-1$. In both cases $g_{11}=g_{22}=1.0, g_{12}=0.6$, $V=2.3$, and $t=160$.
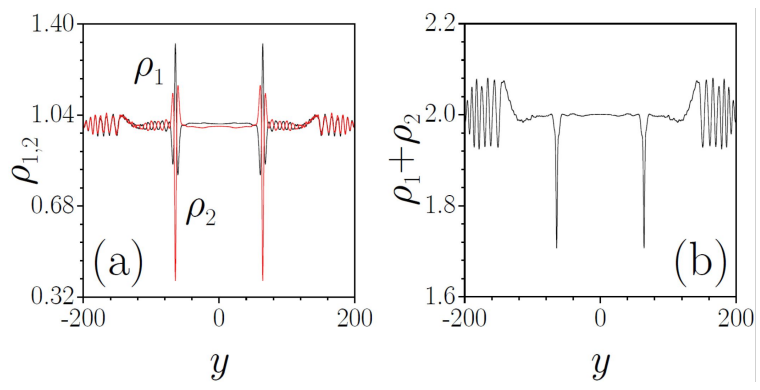

Figure 2. (Color online.) The distributions of densities in two components (a) and total density (b) along the $y$ axis at $x=100, t=160$ [these distributions correspond to dashed lines in Fig. 1(b)].

with time-dependent breather solutions of the associated nonlinear evolution equations.

More detailed structure of the wave pattern generated by the polarized obstacle can be seen in Fig. 2 representing the density distributions along $y$ axis at fixed value of $x$ coordinate. The oblique breather can be represented as a stationary spatially modulated nonlinear wave with counter-phase nonlinear oscillations of the condensate components. When its envelope is much narrower than the distance between two Mach cones, the space between the oblique breather and the density ship waves is occupied by the polarization ship waves clearly visible in Figs. 1 and 2. The parameters of the oblique breather are determined by the parameters of the incoming flow and those of the obstacle. It is worth noticing that the complete cancelation of oscillations of the components in the total density distribution occurs only if $g_{11}=g_{22}$, otherwise the linear eigenmodes correspond to a mixture of pure density and polarization waves (see 


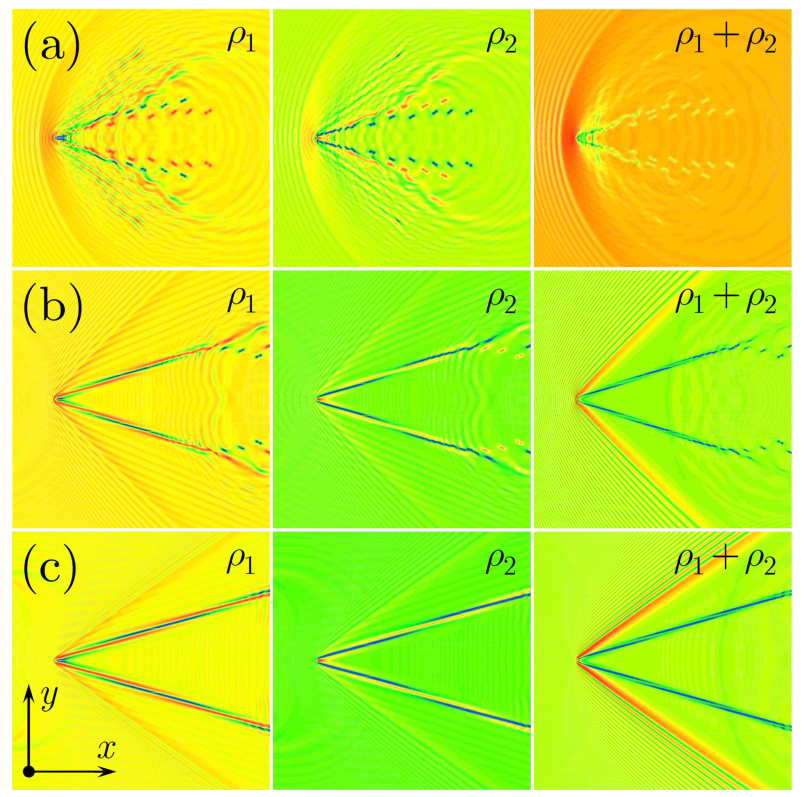

Figure 3. (Color online.) Distributions of the densities of the first (left column) and second (central column) components, as well as of total density (right column) at $t=160$ for the velocity of the flow $V=1.3(\mathrm{a}), V=1.9(\mathrm{~b})$, and $V=2.3$ (c). In all cases $g_{11}=g_{22}=1.0, g_{12}=0.6$.

[25]). At the same time, the wave pattern remains qualitatively the same for small enough difference $g_{11}-g_{22}$. The most important control parameter is the incoming flow velocity $V$. In Fig. 3 we illustrate the modifications in the wave pattern generated by the flow past a polarized obstacle with growth of $V$. If $V=1.3$, then the breather is absolutely unstable and cannot be formed by the flow, and instead the vortex streets are generated. If $V=1.9$, then a clearly resolvable breather is formed and further increase of velocity to $V=2.3$ changes only its inclination angle with respect to the direction of the flow, but does not change essentially its parameters. We suppose that this transition from a non-stationary vortex emission to a stationary formation of oblique breather is physically similar to the transition from absolute instability of oblique dark solitons to their convective instability studied in [12, 13, 26, for the one-component BEC flow.

Analytical theory.-Although the exact breather solutions of the coupled system of GP equations (1) are unknown, we develop here the approximate theory for the small-amplitude breathers which explains with reasonable accuracy the observed features of new wave structures. To this end, we consider a $1 \mathrm{D}$ version of the system (1) which describes waves propagating along the $x^{\prime}$ axis. For separation of density and polarization modes it is convenient to introduce a spinor representation of the field variables 27 .

$$
\left(\begin{array}{l}
\psi_{1} \\
\psi_{2}
\end{array}\right)=\sqrt{\rho} e^{i \Phi / 2} \chi=\sqrt{\rho} e^{i \Phi / 2}\left(\begin{array}{c}
\cos \frac{\theta}{2} e^{-i \phi / 2} \\
\sin \frac{\theta}{2} e^{i \phi / 2}
\end{array}\right),
$$

where $\Phi$ has the meaning of the velocity potential of the in-phase motion; the angle $\theta$ is the variable describing the relative density of the two components $\left(\cos \theta=\left(\rho_{1}-\rho_{2}\right) / \rho\right)$ and $\phi$ is the potential of the relativecounter-phase motion. Correspondingly, the densities of the condensate components are given by $\rho_{1}=$ $\rho \cos ^{2}(\theta / 2), \quad \rho_{2}=\rho \sin ^{2}(\theta / 2)$. In the uniform quiescent state of $\mathrm{BEC}$ with equal densities in the components we can take $\theta=\theta_{0}=\pi / 4$ and then the small-amplitude waves correspond to small variations of the relative density variable $\theta^{\prime} \equiv \theta-\theta_{0}$ and small in-phase $U=\Phi_{x^{\prime}}$ and counter-phase $v=\phi_{x^{\prime}}$ velocities. The perturbation theory 25] for polarization waves with account of small dispersion and weak nonlinearity yields then the evolution equation which in the case when $g_{11}=g_{22} \neq g_{12}$ has the form of $\mathrm{mKdV}$ equation for $\theta^{\prime}$

$$
\theta_{t}^{\prime}+c_{p} \theta_{x^{\prime}}^{\prime}-\frac{3 c_{p}\left(9 g_{11}-g_{12}\right)}{8 g_{12}} \theta^{\prime 2} \theta_{x^{\prime}}^{\prime}-\frac{1}{8 c_{p}} \theta_{x^{\prime} x^{\prime} x^{\prime}}^{\prime}=0 .
$$

If its solution is found, then the other field variables are expressed in terms of $\theta^{\prime}$ by the formulas

$\rho=\rho_{0}-\frac{3 c_{p}^{2}}{2 g_{12}} \theta^{\prime 2}, \quad U=-\frac{c_{p}\left(3 g_{11}+g_{12}\right)}{2 g_{12}} \theta^{\prime 2}, \quad v=2 c_{p} \theta^{\prime}$.

The mKdV equation (5) has a variety of solutions, among which there is the one-dimensional breather solution, first presented in 28, which in our notations can be written as

$$
\theta^{\prime}=-\frac{2}{c_{p}} \sqrt{\frac{2 g_{12}}{9 g_{11}-g_{12}}} \frac{\partial}{\partial x^{\prime}} \arctan \left(\frac{\eta \cos \left(\Theta_{1}+\beta_{1}\right)}{\xi \cosh \left(\Theta_{2}+\beta_{2}\right)}\right),
$$

where

$$
\begin{aligned}
& \Theta_{1}=2 \xi\left(x^{\prime}-c_{p} t\right)-\xi\left(\xi^{2}-3 \eta^{2}\right) t / c_{p}, \\
& \Theta_{2}=2 \eta\left(x^{\prime}-c_{p} t\right)-\eta\left(3 \xi^{2}-\eta^{2}\right) t / c_{p}, \\
& \xi=\kappa \cos q, \quad \eta=\kappa \sin q, \\
& \beta_{1}=p-q, \quad \beta_{2}=\ln (2 \kappa \tan q / a)
\end{aligned}
$$

and $p, q, \kappa, a$ are free parameters. This solution gives an approximate description of the oblique breather pattern found above numerically when it is transformed to the appropriate reference frame and the parameters are chosen in a proper way. The solution (7) is written in the reference frame associated with a quiescent condensate, where the breather propagates with the envelope velocity $V_{b}=c_{p}+\left(3 \xi^{2}-\eta^{2}\right) /\left(2 c_{p}\right)$ and the carrier wave velocity $V_{c}=c_{p}+\left(\xi^{2}-3 \eta^{2}\right) /\left(2 c_{p}\right)$ along the $x^{\prime}$ axis with the breather location line parallel to the $y^{\prime}$ axis. We must transform it to the reference frame with the obstacle located at the axes origin, the condensate's flow 

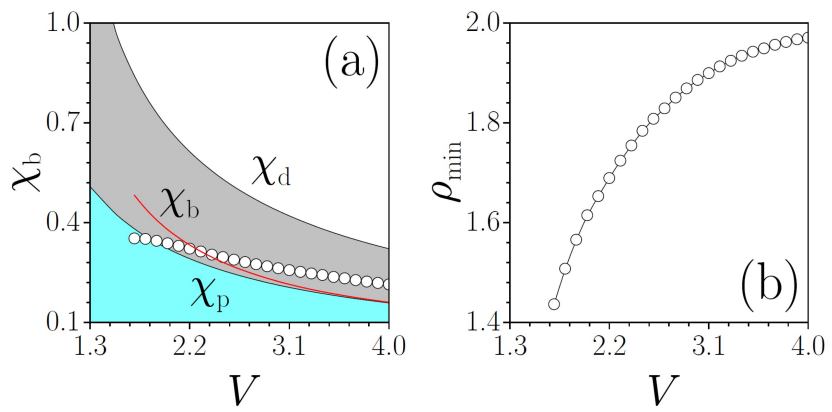

Figure 4. (Color online.) The inclination angle $\chi$ (a) and depth $\rho_{\text {min }}=\rho_{0}-\Delta \rho$ (b) of the oblique breather generated by the flow of condensate past the polarized obstacle versus flow velocity $V$ at $g_{11}=g_{22}=1.0, g_{12}=0.6$. In (a) the line with circles shows numerical results, while red solid line shows analytical prediction (9) for the inclination angle. Blue region corresponds to the inner Max cone, while the region between inner and outer Max cones is shown grey.

velocity directed along the $x$ axis, and the breather location line inclined with respect to the $x$ axis at some angle $\chi_{b}$ chosen in such a way that the breather becomes a stationary structure in the new reference frame. The same transformation must compensate both the envelope velocity and the carrier wave velocity, $V_{b}=V_{c}$, what gives $q=\pi / 4$. Besides that, these two velocities must be compensated by the projection of the flow velocity $V \sin \chi_{b}$ on the $x^{\prime}$ axis. That can be realized only at $c_{p}+\kappa^{2} /\left(2 c_{p}\right)=V \sin \chi_{b}$. After these transformations the phases $\Theta_{1}$ and $\Theta_{2}$ must be replaced by $\Theta_{1}=\Theta_{2}=\sqrt{2} \kappa \cos \chi_{b}\left(y \pm \tan \chi_{b} \cdot x\right)$ and, as a result, the solution (7) transforms into the distribution of $\theta^{\prime}$ in the $(x, y)$-plane. Substitution of $\theta^{\prime}(x, y)$ into Eqs. (8) yields the distributions of the other field variables.

It is convenient to express the parameter $\kappa$ in terms of the maximal amplitude of the density envelope $\Delta \rho \equiv$ $\left|\rho-\rho_{0}\right|=24 \kappa^{2} /\left(9 g_{11}-g_{12}\right)$. As a result, we get, with account of the expression $c_{p}^{2}=\rho_{0}\left(g_{11}-g_{12}\right) / 2$ which is valid if $g_{11}=g_{22}$, a useful relation between the breather angle $\chi_{b}$ and the maximal envelope amplitude $\Delta \rho$ :

$$
\sin \chi_{b}=\frac{1}{M_{p}}\left(1+\frac{9 g_{11}-g_{12}}{24\left(g_{11}-g_{12}\right)} \cdot \frac{\Delta \rho}{\rho_{0}}\right) .
$$

Importantly, this formula predicts that the oblique breathers are located outside the polarization Mach cone defined by Eq. (3). Another important relation is given by the dependence of the inverse width $w=4 \eta$ of the breather on its amplitude $\Delta \rho$ :

$$
w=\sqrt{\left(9 g_{11}-g_{12}\right) \Delta \rho / 3} .
$$

Numerically found dependence of the breather angle $\chi_{b}$ as a function of $V$ is shown in Fig. 4(a) by circles. As one can see, it agrees with qualitative prediction that oblique

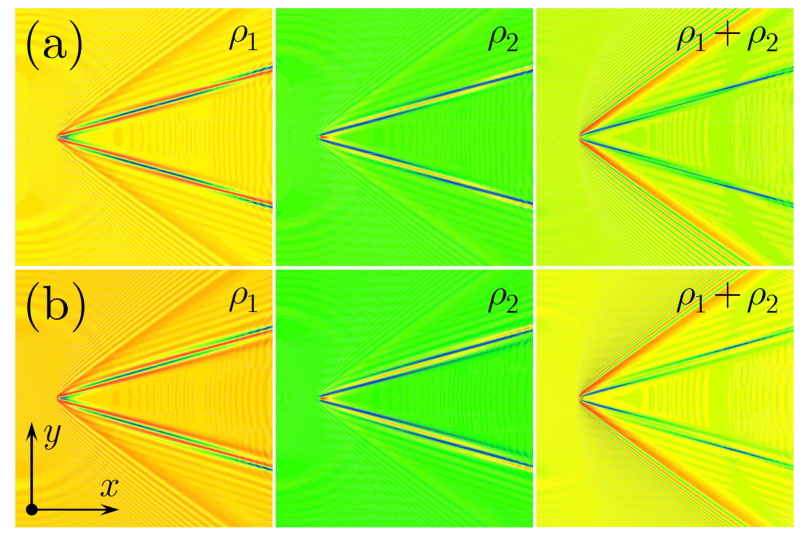

Figure 5. (Color online.) Density distributions generated by the polarized obstacle at (a) $g_{11}=1.1, g_{22}=0.9$ and (b) $g_{11}=1.2, g_{22}=0.8$. In both cases $V=2.3, t=160$, and $g_{12}=0.6$.

breathers are located outside the polarization Mach cone. For comparison with the analytical formula (9) we determined numerically the minimal density $\rho_{\min }=\rho_{0}-\Delta \rho$ in the breather as a function of $V$ which is plotted in Fig. 4(b). Substitution of corresponding values of $\Delta \rho$ into Eq. (9) yields the dependence of $\chi_{b}$ on $V$ which is shown in Fig. 4(a) by a red line. It perfectly agrees with numerical values of $\chi_{b}$ at velocities close to $V \approx 2$, however, the theoretical dependence deviates from the numerical one for $V \gtrsim 3$. We explain this disagreement by the fact that at $V \simeq 3$ the breather's width given by Eq. 10 is of the same order as the distance between the two Mach cones and, consequently, the breather cannot be considered as a structure well separated from other excitations - in this case the interaction of the breather with the ship wave leads to deviations of the breather's parameters from those calculated in the frames of perturbation theory. Another consequence of the interaction of the breather with the ship wave, slowly changing along the Mach cone, is the finite lengths of wavecrests in the carrier wave.

Arbitrary nonlinearity constants. - The above theory was developed for the case when $g_{11}=g_{22}$. Nevertheless, it is of considerable interest to study what happens if $g_{11} \neq g_{22}$. Corresponding results obtained by direct solution of Eqs. (1) are presented in Fig. 5. One can see that now the counter-phase oscillations of the densities of components do not compensate each other and the ship wave pattern becomes clearly visible in the distribution of the total density. With increase of the difference $g_{11}-g_{22}$ the width of the oblique breathers also increases but qualitatively the whole wave pattern remains the same.

Summarizing, we have predicted that new nonlinear structures - oblique breathers - can be generated by a flow of two-component condensate past polarized obstacles. 
[1] T. Frisch, Y. Pomeau, and S. Rica, Phys. Rev. Lett. 69, 1644 (1992).

[2] T. Winiecki, J. F. McCann, and C. S. Adams, Phys. Rev. Lett. 82, 5186 (1999).

[3] N. G. Berloff and P. H. Roberts, J. Phys. A: Math. Gen. 33, 4025 (2000).

[4] S. Rica, Physica D 148, 221 (2001).

[5] C.-T. Pham, C. Nore, and M.-É. Brachet, Physica D 210, 203 (2005).

[6] D. L. Kovrizhin and L. A. Maksimov, Phys. Lett. A 282, 421 (2001).

[7] G. E. Astrakharchik and L. P. Pitaevskii, Phys. Rev. A 70, 013608 (2004).

[8] I. Carusotto, S. X. Hu, L. A. Collins, and A. Smerzi, Phys. Rev. Lett. 97, 260403 (2006).

[9] Yu. G. Gladush, G. A. El, A. Gammal, and A. M. Kamchatnov, Phys. Rev. A 75, 033619 (2007).

[10] Y. S. Kivshar and B. Luther-Davies, Phys. Rep. 298, 81 (1998).

[11] G. A. El, A. Gammal, and A. M. Kamchatnov, Phys. Rev. Lett. 97, 180405 (2006).

[12] A. M. Kamchatnov and L. P. Pitaevskii, Phys. Rev. Lett. 100, 160402 (2008).

[13] A. M. Kamchatnov and S. V. Korneev, Phys. Lett. 375, 2577 (2011).

[14] A. Amo, S. Pigeon, D. Sunvitto, V. G. Sala, R. Hivet, I. Carusotto, F. Pisanello, G. Leménager, R. Houdré, E. Giacobino, C. Ciuti, and A. Bramati, Science 332, 1167 (2011).

[15] G. Grosso, G. Nardin, F. Morier-Genoud, Y. Léger, and B. Deveaud-Plédran, Phys. Rev. Lett. 107, 245301
(2011).

[16] C. J. Myatt, E. A. Burt, R. W. Grist, E. A. Cornell, and C. E. Wiemann, Phys. Rev. Lett. 78, 586 (1997).

[17] D. M. Stamper-Kurn, M. R. Andrews, A. P. Chikkatur, S. Inouye, H.-J. Miesner, J. Stenger, and W. Ketterle, Phys. Rev. Lett., 80, 2027 (1998).

[18] J. Kasprzak, M. Richard, S. Kundermann, A. Baas, P. Jeambrun, J. M. J. Keeling, F. M. Marchetti, M. H. Szymanska, R. André, J. L. Staehli, V. Savona, P. B. Littlewood, B. Deveaud, and L. S. Dang, Nature, 443, 409 (2006).

[19] R. Balili, V. Hartwell, D. Snoke, L. Pfeiffer, and K. West, Science, 316, 5827 (2007).

[20] D. J. Frantzeskakis, J. Phys. A: Math. Theor. 43, 213001 (2010).

[21] I. Carusotto and C. Ciuti, Rev. Mod. Phys. 85, 299 (2013).

[22] H. Flayac, D. D. Solnyshkov, and G. Malpuech, Phys. Rev. B 83, 193305 (2011).

[23] R. Hivet, H. Flayac, D. D. Solnyshkov, D. Tanese, T. Boulier, D. Andreoli, E. Giacobino, J. Bloch, A. Bramati, G. Malpuech, and A. Amo, Nature Phys. 8, 724 (2012).

[24] Yu. G. Gladush, A. M. Kamchatnov, Z. Shi, P. G. Kevrekidis, D. J. Frantzeskakis, and B. A. Malomed, Phys. Rev. A 79, 033623 (2009).

[25] A. M. Kamchatnov, Y. V. Kartashov, P.-É. Larré, and N. Pavloff, arXiv:1308.0784 (2013).

[26] M. A. Hoefer and B. Ilan, Multiscale Model. Simul., 10, 306 (2012).

[27] K. Kasamatsu, M. Tsubota, and M. Ueda, Phys. Rev. A, 71, 043611 (2005).

[28] M. Wadati, J. Phys. Soc. Japan, 34, 1289 (1973). 